\title{
Lhermitte-Duclos Disease: Case Report and Literature Review
}

\author{
Brajesh Kumar ${ }^{1}$, Samrendra Kumar Singh ${ }^{2}$, Om Prakash Gupta ${ }^{3}, \mathrm{KM} \mathrm{Jha}^{4}$
}

\begin{abstract}
Lhermitte-Duclos disease (LDD) is a neurological disorder caused by a hamartomatous lesion within the cerebellum. Clinically, LDD is often associated with symptoms of the space-occupying lesion due to increased intracranial pressure, hydrocephalus, and focal neurological deficit. This was a case of a 36-year-old woman who presented with progressive headache and difficulty in swallowing liquids. Magnetic resonance imaging demonstrated a right cerebellar mass lesion with the characteristic "tiger-striped appearance". There was an inferior displacement of the right cerebellar tonsil. She underwent ventriculoperitoneal (VP) shunt and followed by partial resection of the tumor to decrease the mass effect. Her headache gradually improved and she was able to swallow liquids by the time of discharge.
\end{abstract}

Keywords: Dysplastic gangliocytoma, Headache, Lhermitte-Duclos disease.

International Journal of Head and Neck Surgery (2021): 10.5005/jp-journals-10001-1424

\section{INTRODUCTION}

Lhermitte-Duclos disease (LDD), also known as dysplastic gangliocytoma of the cerebellum, is a rare disease with slowly progressive unilateral cerebellar mass lesion, characterized by a slowly progressive unilateral cerebellar mass lesion. ${ }^{1,2}$ LhermitteDuclos disease affects adults of both sexes. It usually presents with symptoms caused by a mass effect caused by growing cerebellar mass, e.g., headache, vomiting, cerebellar dysfunction, occlusive hydrocephalus, and cranial nerve palsies. ${ }^{1,2}$ Clinical occurrence is most frequent among young adults, but individuals of all ages can be affected. ${ }^{1}$

We describe herein a rare case involving a female patient with LDD and subsequently discuss the management of LDD in such patients.

\section{Case Description}

We describe here this rare case in a 36-year-old woman who presented in OPD with progressive headache and progressive difficulty in swallowing liquids indicating lower cranial nerve palsy. She did not have any visual difficulties, hearing difficulties, weakness or thinning of any part of her limbs, difficulty in walking or frequent falls, swaying, and sensory symptoms. Motor and sensory examinations were normal. Gag reflex was diminished. Cerebellar, gait, and higher mental function examinations were normal. Computed tomography (CT) and magnetic resonance imaging (MRI) brain with contrast was ordered as an additional investigation. CT scan showed low-density mass in the right cerebellar hemisphere (Fig. 1). Magnetic resonance imaging revealed a sharply defined mass in the right cerebellar hemisphere. The mass appeared hyperintense on diffusion-weighted imaging (Fig. 2) and hypointense on both fluid-attenuated inversion recovery imaging and T1-weighted imaging (Figs 2 and 3). The characteristic "tiger-striped appearance" was visible on T2-weighted imaging (Figs 4 and 5).

Mass effect was evident at the medulla oblongata, accompanied by inferior displacement of the right cerebellar tonsil which was

\footnotetext{
${ }^{1-4}$ Department of Neurosurgery, Indira Gandhi Institute of Medical Sciences, Patna, Bihar, India
}

Corresponding Author: Brajesh Kumar, Department of Neurosurgery, Indira Gandhi Institute of Medical Sciences, Patna, Bihar, India, Phone: +91 9674200878, e-mail: drbrajeshkumar@icloud.com

How to cite this article: Kumar B, Singh SK, Gupta OP, et al. LhermitteDuclos Disease: Case Report and Literature Review. Int J Head Neck Surg 2021;12(2):65-70.

Source of support: Nil

Conflict of interest: None

likely the cause of the headache and lower cranial nerve deficit in this case.

We planned for ventriculoperitoneal shunt (VP shunt) followed by decompression of the lesion through midline sub-occipital approach. The right cerebellar hemisphere was pale and enlarged

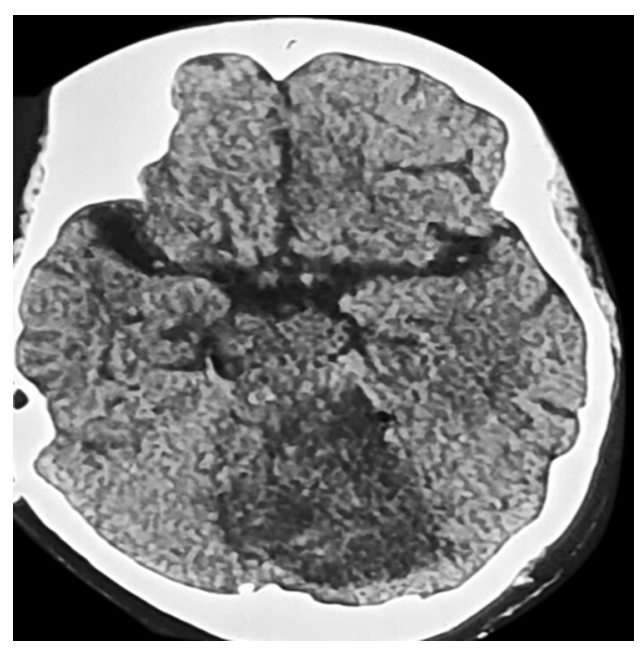

Fig. 1: CT scan showing hypodense mass in right cerebellar region

\footnotetext{
(c) The Author(s). 2021 Open Access This article is distributed under the terms of the Creative Commons Attribution 4.0 International License (https:// creativecommons.org/licenses/by-nc/4.0/), which permits unrestricted use, distribution, and non-commercial reproduction in any medium, provided you give appropriate credit to the original author(s) and the source, provide a link to the Creative Commons license, and indicate if changes were made. The Creative Commons Public Domain Dedication waiver (http://creativecommons.org/publicdomain/zero/1.0/) applies to the data made available in this article, unless otherwise stated.
} 
with widened folia but margins to the mass lesion were not easily defined (Fig. 2). Partial resection of the mass to solve tonsillar herniation was thus performed (Fig. 6), and subsequent histological

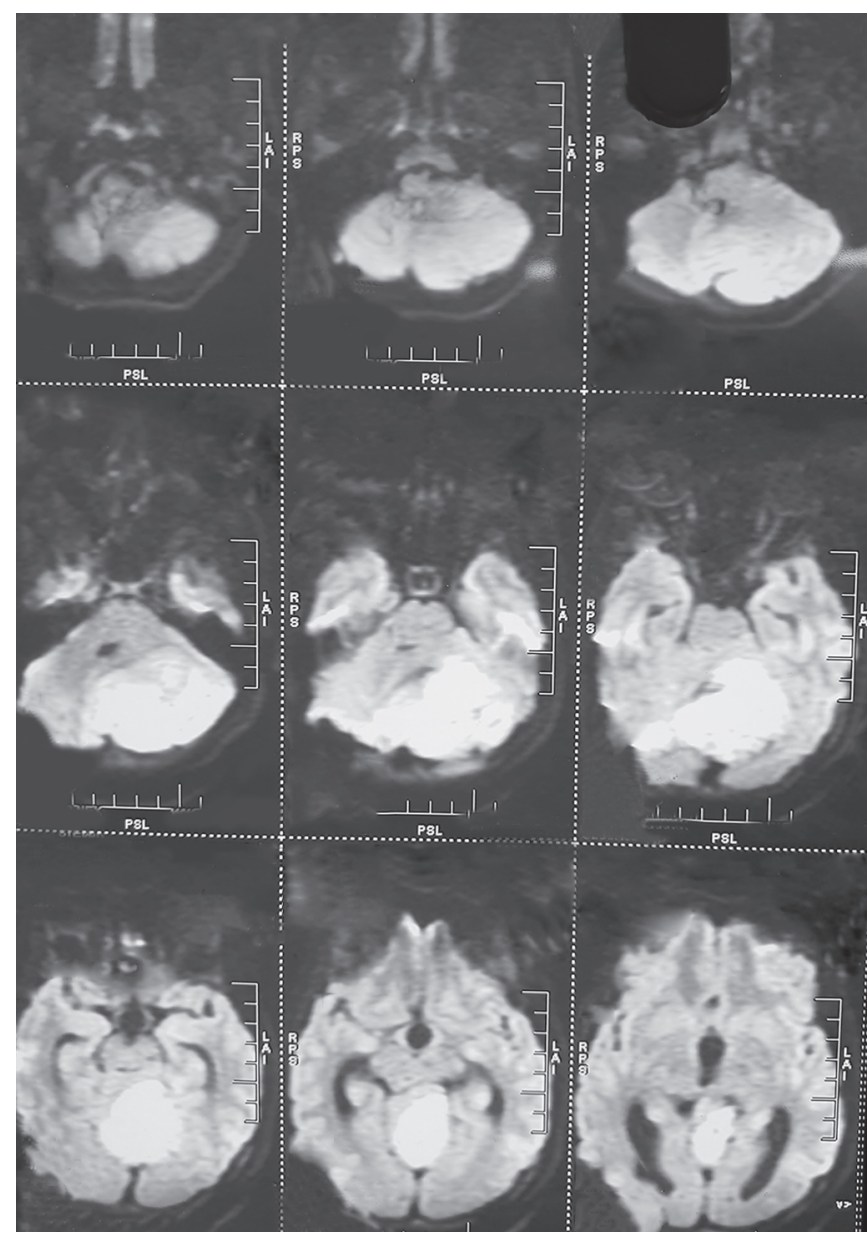

Fig. 2: MRI axial DW1 examination confirmed the diagnosis of LDD. The molecular layer was enlarged with dysplastic ganglion-like cells, hypertrophied granular layer, and increased numbers of small blood vessels within the molecular layer were detected by hematoxylin and eosin staining (Figs 7 and 8).

\section{Discussion}

Lhermitte-Duclos disease is a rare disease that does not have a clear nature and pathogenesis. ${ }^{1,2}$ More than 200 cases have been reported in the literature. ${ }^{3}$ Most of the cases occurred in adults in the third and fourth decades of life similar to our case, without any sex predilection. ${ }^{1,2}$ Lesions found in the elderly have tendencies toward male predominance, hypervascularity, and low rates of association with Cowden's disease (CD) and regrowth after surgery. In most of the studies, patients presented with symptoms and signs of raised intracranial pressure were managed with partial resection and with or without VP shunt depending on the presence of hydrocephalus. Lhermitte-Duclos disease may be accompanied by other malignant tumors, e.g., thyroid carcinoma, breast carcinoma, endometrial carcinoma, prostate carcinoma, and glioblastoma. ${ }^{1,2}$ On MRI, none of the studies showed contrast enhancement in LDD. Lesions were hyperintense on T2-weighted imaging and hypointense on T1-weighted imaging giving rise to a "tiger-striped" appearance. ${ }^{1-4}$ Complete excision of the hypertrophied lesion is the treatment of choice. $^{1-3,5-7}$ There has been a report of regrowth or malignant transformation following subtotal resection. ${ }^{8,9}$ Many authors have recommended gross total removal in young adults, even when the patient is asymptomatic. ${ }^{8}$ Most of the times it is very difficult to perform complete excision because the lesion cannot be differentiated from normal cerebellar tissue. ${ }^{2,3,10,11}$ Extensive resection risks include neurological deficits such as cerebellar mutism. ${ }^{5}$ Almost all reported patients were symptomatic and underwent partial resection to reduce the mass effect. We also could not define the margins of the mass and partial resection was performed. Headache and lower cranial nerve palsy improved without complication (Fig. 9). Moreover, the regrowth/malignant

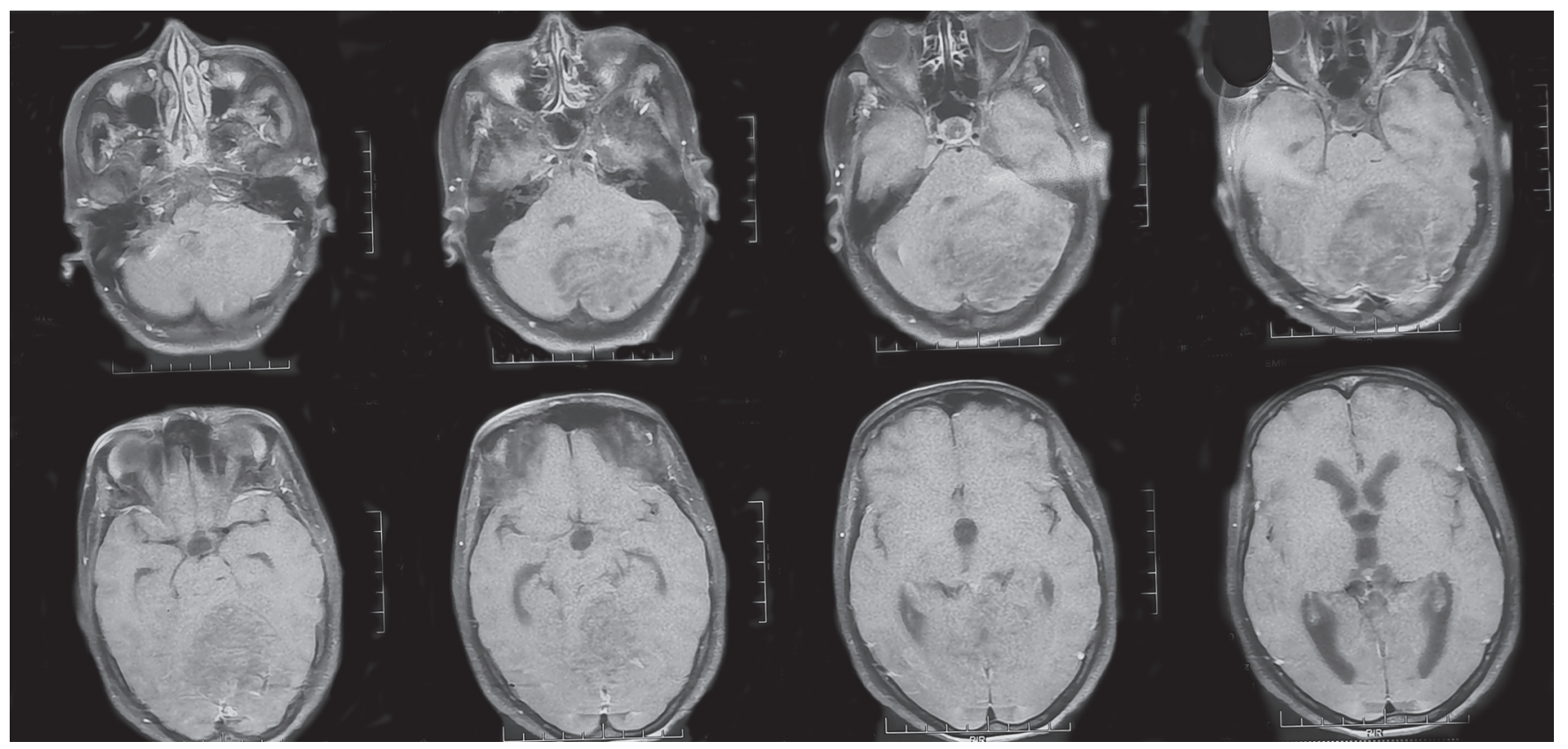

Fig. 3: MRI brain axial flair 


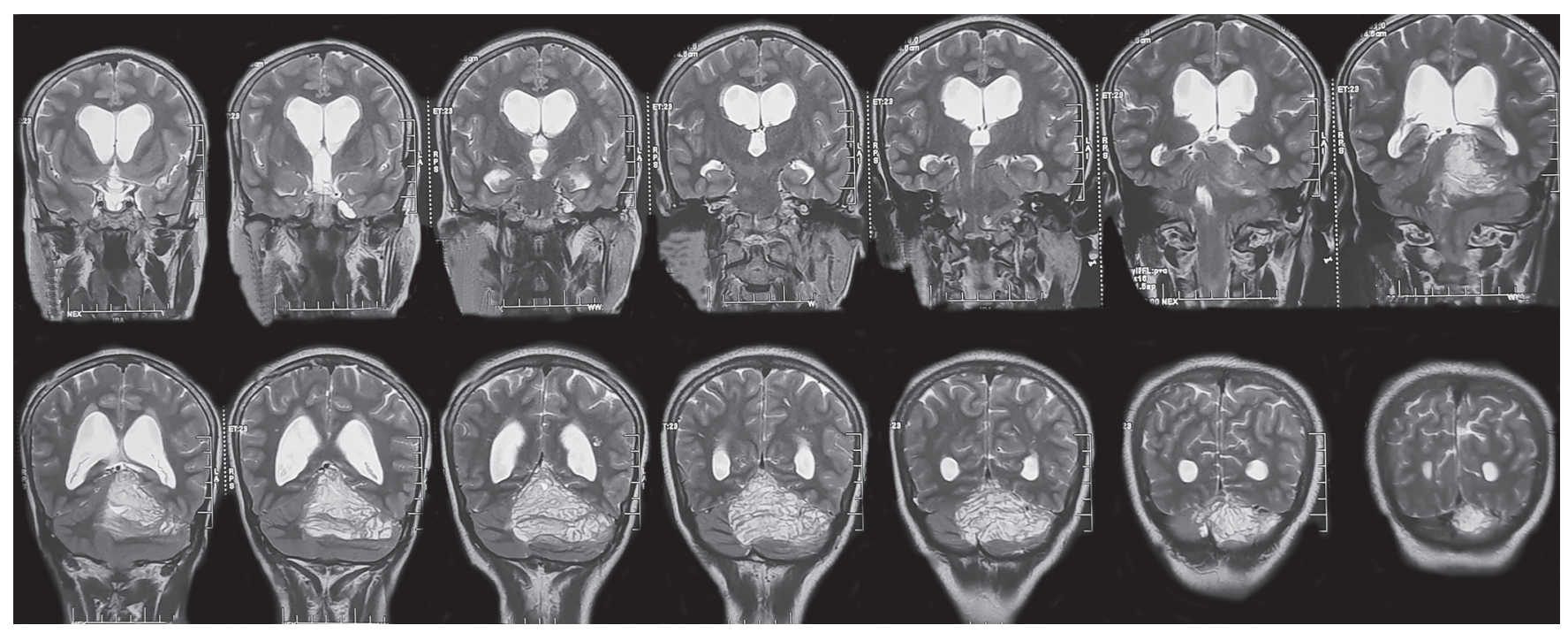

Fig. 4: MRI brain T2 coronal "tiger-stripped appearance"

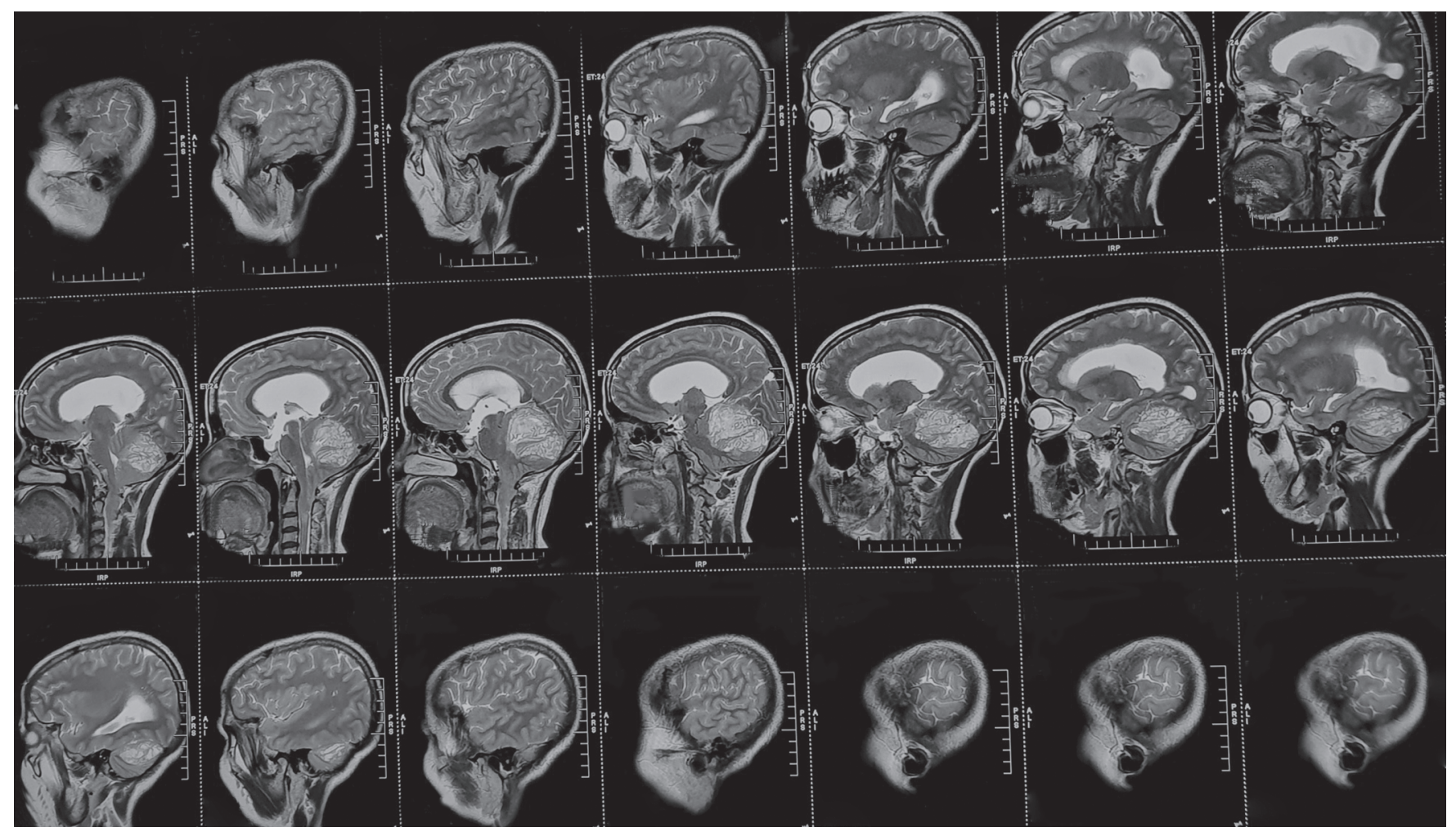

Fig. 5: MRI brain T2 sagittal

transformation of the tumor was reported in a very small number. Partial resection to reduce mass effect can thus be chosen, because the lesion is categorized as a World Health Organization grade I tumor. If the mass is accidentally found, a conservative strategy can be chosen with close follow-up examination.

\section{CONCLUSION}

Lhermitte-Duclos disease is a rare disease having a benign course and pathological findings. In symptomatic LDD, we recommend partial resection to reduce the mass effect.

\section{References}

1. NowakDA, Trost HA. Lhermitte-Duclos disease (dysplasticcerebellar gangliocytoma): a malformation, hamartoma or neoplasm? Acta Neurol Scand 2002;105(3):137-145. DOI: 10.1034/j.16000404.2002.1r127.x.

2. Andres RH, Guzman R, Weis J, et al. Lhermitte-Duclos disease with atypical vascularization - case report and review of the literature. Clin Neuropathol 2009;28(03):83-90. DOI: 10.5414/NPP28083.

3. Ishizaki K, Daita G, Yonemasu Y, et al. Hypervascularity in LhermitteDuclos disease-case report. Neurol Med Chir (Tokyo) 1997;37(5):403406. DOI: 10.2176/nmc.37.403. 


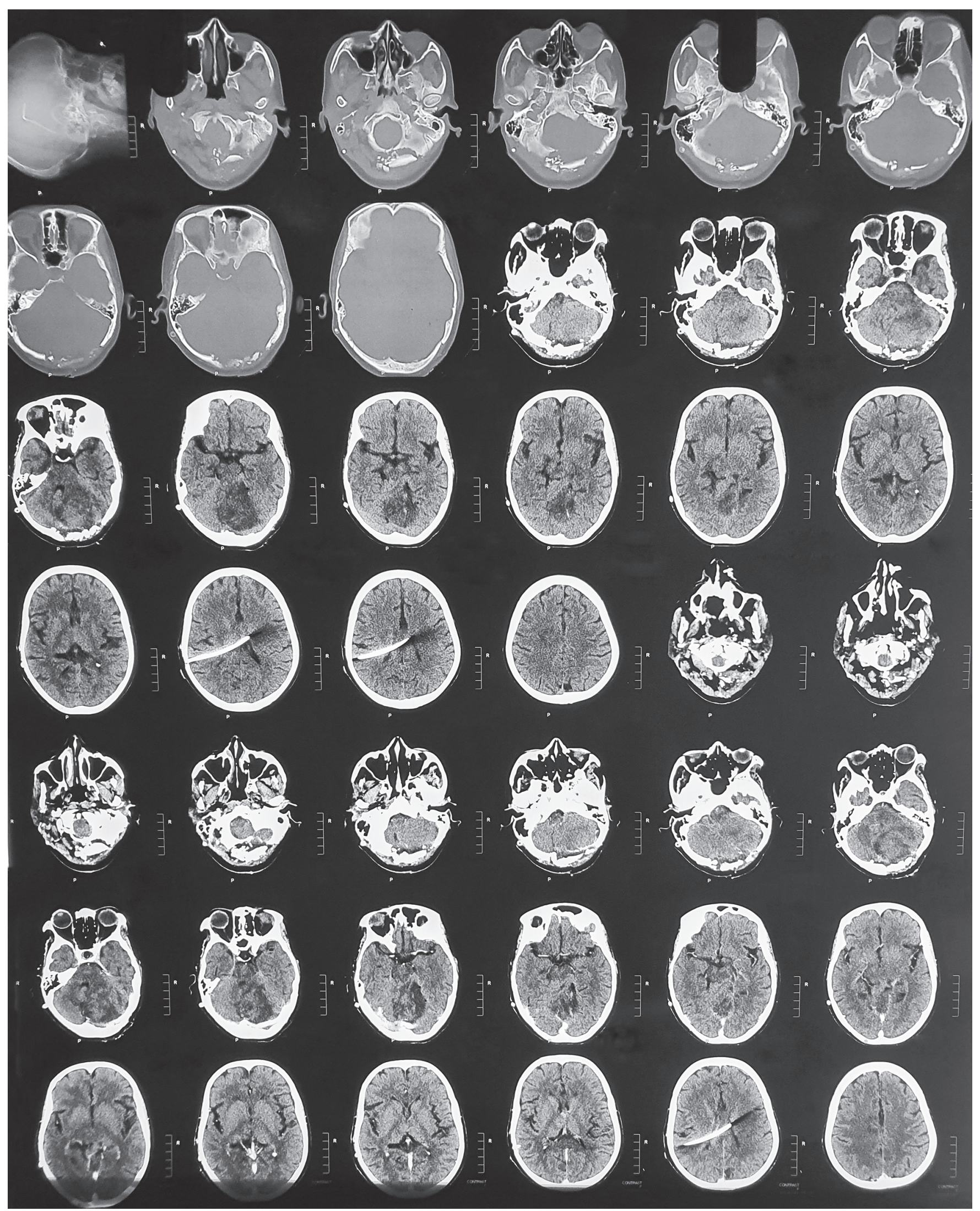

Fig. 6: Postoperative CT scan 


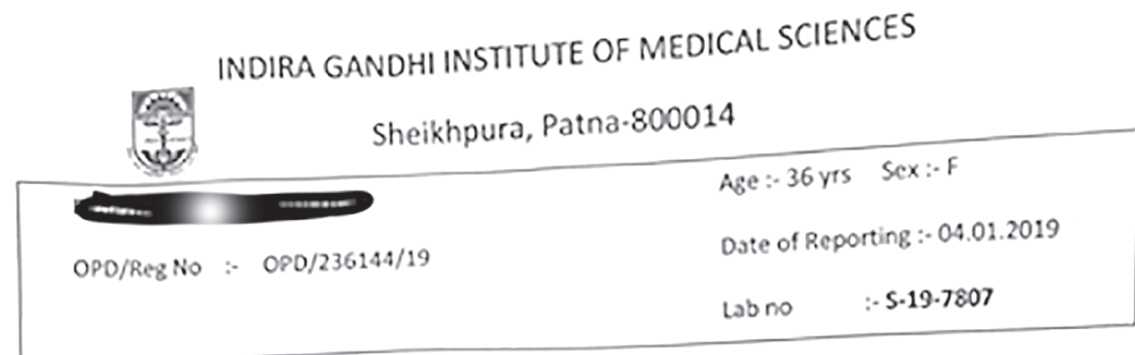

Histopathology Report

INVESTIGATION :- HPE of left cerebellar SOL.

GROSS :- Rereived multiple grey white soft tissue picces amounting to $3 c c$

Microscopy :- Section shows diffuse enlargement of internal granular layer and moleduclar layers with presence of dysplastic ganglion cells along with normal cerebellar tissue.

IMPRESSION :- Features are consistent with dysplastic gangliocytoma (Ihermite duclos syndiome).

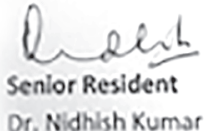

Or. Nidhlsh Kumar

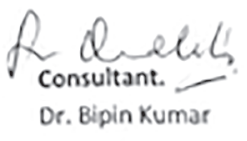

Dr. Bipin Kumar

Fig. 7: Histopathology report LD syndrome
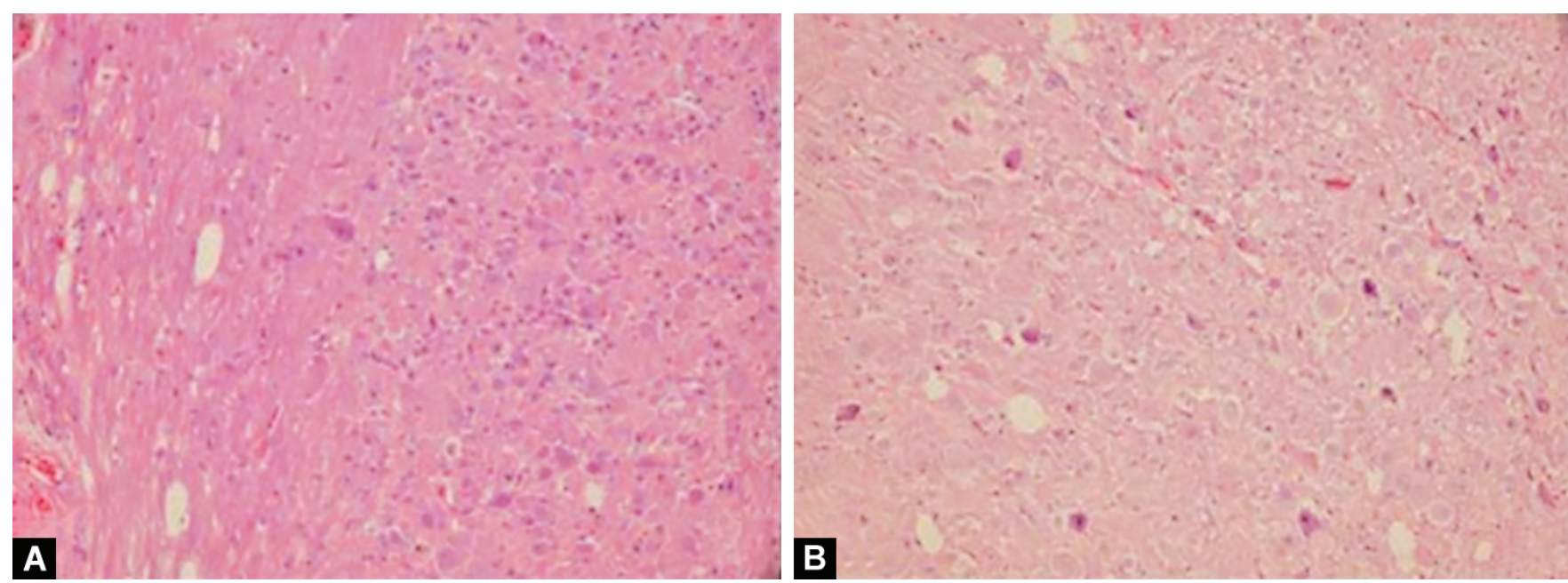

Figs $8 \mathrm{~A}$ and B: Histopathology slide of Lhermitte-Duclos syndrome 


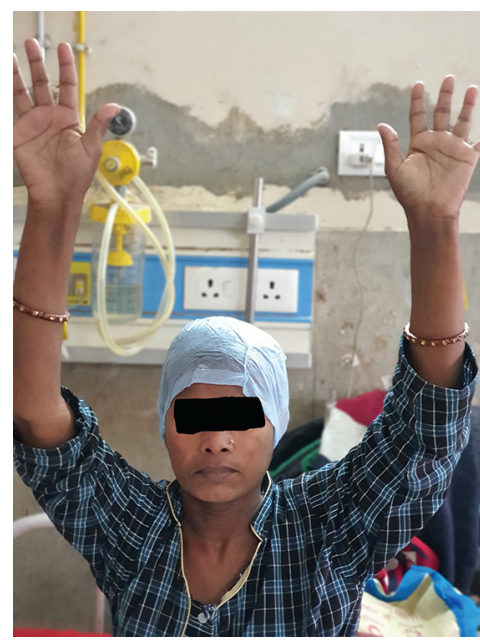

Fig. 9: Postoperative patient image

4. Moonis G, Ibrahim M, Melhem ER. Diffusion-weighted MRI in Lhermitte-Duclos disease: report of two cases. Neuroradiology 2004;46(5):351-354. DOI: 10.1007/s00234-004-1190-6.
5. Afshar-Oromieh A, Linhart $H$, Podlesek $D$, et al. Postoperative cerebellar mutism in adult patients with Lhermitte-Duclos disease. Neurosurg Rev 2010;33(4):401-408. DOI: 10.1007/s10143-0100278-1.

6. Goto $Y$, Hashimoto N, Okita $Y$, et al. A surgically treated case of Lhermitte-Duclos disease with a precise natural history and high uptake of FDG on PET. J Neurooncol 2010;97(3):445-450. DOI: 10.1007/ s11060-009-0042-y.

7. Zou YH, Cao YQ, Yue ZJ, et al. Unusual posterior fossa mass caused by Lhermitte-Duclos disease with no symptoms in adults. Br J Neurosurg 2011;26(1):99-101. DOI: 10.3109/02688697.2011.591851.

8. Inoue T, Nishimura S, Hayashi N, et al. Ectopic recurrence of dysplastic gangliocytoma of the cerebellum (Lhermitte-Duclos disease): a case report. Brain Tumor Pathol 2007;24(1):25-29. DOI: 10.1007/s10014006-0211-z.

9. Takei H, Dauser R, Su J, et al. Anaplastic ganglioglioma arising from a Lhermitte-Duclos-like lesion. Case report. J Neurosurg 2007;107:137142.

10. Nakagawa T, Maeda M, Kato M, et al. A case of Lhermitte-Duclos disease presenting high FDG uptake on FDG-PET/CT. J Neurooncol 2007;84(2):185-188. DOI: 10.1007/s11060-007-9355-x.

11. Yang MS, Kim CH, Cheong JH, et al. Lhermitte-Duclos disease presenting with hydrocephalus. Acta Neurochir Suppl 2012;113: 161-165. 